\title{
2-Azolylchromone Derivatives as Potent and Selective Inhibitors of Monoamine Oxidases A and B
}

\author{
Koichi Takao, ${ }^{*}$ Takayuki Saito, Daisuke Chikuda, and Yoshiaki Sugita \\ Laboratory of Bioorganic Chemistry, Department of Pharmaceutical and Health Sciences, Faculty of Pharmaceutical \\ Sciences, Josai University; 1-1 Keyaki-dai, Sakado, Saitama 350-0295, Japan. \\ Received June 30, 2016; accepted July 24, 2016
}

A series of 2-azolylchromone derivatives were synthesized and their monoamine oxidase (MAO) A and $B$ inhibitory activities were evaluated. Of the synthesized compounds, compounds $1 \mathrm{~b}, 2 \mathrm{~b}, 4 \mathrm{a}-\mathrm{c}, 5 \mathrm{~b}$ and 7b showed potent inhibitory activities against MAO-A ( $\mathrm{IC}_{50}$ values, 1b: $0.32 \mu \mathrm{M} ; 2 \mathrm{~b}: 0.14 \mu \mathrm{M} ; 4 \mathrm{a}: 0.11 \mu \mathrm{M}$; 4b: $0.023 \mu \mathrm{M} ; 4 \mathrm{c}: 0.15 \mu \mathrm{M} ; 5 \mathrm{~b}: 0.59 \mu \mathrm{M} ; 7 \mathrm{~b}: 0.19 \mu \mathrm{M}$ ) and 4a, c, 5a, c, 6c and 8c for MAO-B (IC 50 values, 4a: $0.028 \mu \mathrm{M} ; 4 \mathrm{c}: 0.019 \mu \mathrm{M} ; 5 \mathrm{a}: 0.73 \mu \mathrm{M} ; 5 \mathrm{c}: 0.28 \mu \mathrm{M} ; 6 \mathrm{c}: 0.28 \mu \mathrm{M} ; 8 \mathrm{c}: 0.27 \mu \mathrm{M})$. These data suggest that 6-methoxy substitution favors MAO-A inhibition and 7-methoxy substitution favors MAO-B inhibition. In addition, compound 4b was the most potent inhibitor for MAO-A, and compound 4c for MAO-B. This is the first report identifying 2 -azolylchromone derivatives as potent monoamine oxidase inhibitors. These results suggest that the 2-triazolylchromone structure may be a useful scaffold for the design and development of novel monoamine oxidase inhibitors, as evidenced by the activities of $4 \mathrm{a}-\mathrm{c}$ and $5 \mathrm{a}-\mathrm{c}$.

Key words 2-azolylchromone; monoamine oxidase A; monoamine oxidase B; inhibitor; structure-activity relationship; 2-triazolylchromone

4H-1-Benzopyran-4-ones (chromones) are an important class of oxygenated heterocyclic compounds that have attracted the attention of organic chemists and biochemists due to their biological activities. The chromone core structure is found in flavones and isoflavones, which are secondary metabolites that are ubiquitous in nature and especially in the plant kingdom, and are present in notable amounts in several species of plants. The chromone scaffold is the pharmacophore of a large number of bioactive molecules of either natural or synthetic origin. The biological effects of these bioactive molecules include anti-inflammatory, anti-tumor and anti-microbial activities, and inhibitory activities towards cyclooxygenases, kinases, phosphatases, aromatases, acetylcholinesterases, and monoamine oxidases. ${ }^{1,2)}$

The monoamine oxidases $\mathrm{A}$ and $\mathrm{B}$ (EC 1.4.3.4; MAO-A and MAO-B) are flavoenzymes that play an important role in the oxidative degradation of neurotransmitters such as dopamine, serotonin, and epinephrine. MAOs are found in the outer mitochondrial membrane of various mammalian cell types. MAO-A and MAO-B share approximately $70 \%$ sequence identity at the amino acid level and were identified based on their substrate selectivities and inhibitor sensitivities. MAO-A preferentially deaminates serotonin, norepinephrine, and epinephrine and is irreversibly inhibited by clorgyline, whereas MAO-B preferentially deaminates dopamine, $\beta$-phenetylamine, and benzylamine and is irreversibly inhibited by $R$-(-)-deprenyl. MAO inhibitors are important in the treatment of several neurodegenerative diseases. Selective MAO-A inhibitors are used as anti-depressant and anti-anxiety drugs whereas selective MAO-B inhibitors are used to treat Parkinson's disease. ${ }^{3-5)}$

Recently, several research groups have reported chromone derivatives substituted at different positions of the chromone ring and their inhibitory effects towards MAO-A and MAO-B., ${ }^{2,6-12)}$ These reports suggested that structural changes induced by the substitutions on the chromone ring can in- crease the biological activity of the compound.

Azoles such as pyrrole, ${ }^{13)}$ pyrazole, ${ }^{14)}$ indole $^{15)}$ and benzazole ${ }^{16)}$ containing compounds have also been reported as potent MAO inhibitors. We therefore proposed that chromones bearing an azole moiety should display interesting monoamine oxidase inhibitory activities.

In this report we explored promising lead compounds for developing MAO inhibitors by synthesizing a series of 2-azoylchromone derivatives for the first time and investigating their MAO inhibitory activities.

\section{Results and Discussion}

Chemistry The 2-azolylchromone derivatives presented in this study were synthesized by conjugated addition reactions of 3-iodochromone derivatives (III) with azoles according to the method of Sugita and Yokoe ${ }^{17}$ (Chart 1). This required preparation of the 3 -iodochromone derivatives IIIa-c $\left(\mathrm{R}^{1}\right.$, $\mathrm{R}^{2}=\mathrm{H}$ and $\mathrm{OMe}$, respectively). These compounds were synthesized from 2-hydroxyacetophenone derivatives (I) via enaminoketone derivatives (II) according to the method of Vasselin et $a l .{ }^{18)}$ Satisfactory yields were obtained in all cases.

Biological Activity All the synthesized 2-azolylchromone derivatives were evaluated for MAO-A and MAO-B inhibitory activities. As shown in Table 1, different azole modifications and methoxy substitutions on the chromone ring revealed several interesting structure-activity relationships. The MAO-A inhibitory activities of the 2-azolylchromone derivatives were determined, with 7 of the 24 tested 2-azolylchromones displaying various degrees of potency. Compound $\mathbf{4 b}$ was the most potent inhibitor $\left(\mathrm{IC}_{50}=0.023 \mu \mathrm{M}\right)$. Compounds $\mathbf{1 b}, \mathbf{2 b}$, $\mathbf{4 a}, \mathbf{c}, \mathbf{5 b}$ and $\mathbf{7 b}$ showed potent inhibitory activities, with sub-micro molar $\mathrm{IC}_{50}$ values. Interestingly, 6-methoxy substituted compounds, such as $\mathbf{1 b}, \mathbf{2 b}, \mathbf{4 b}, \mathbf{5 b}$ and $\mathbf{7 b}$, also showed potent inhibitory activities against MAO-A. Compounds $\mathbf{3 b}$, $\mathbf{6 b}$ and $\mathbf{8 b}$ showed selectivity for MAO-A compared with the unsubstituted or 7-methoxy substituted 2-azolylchromone de- 


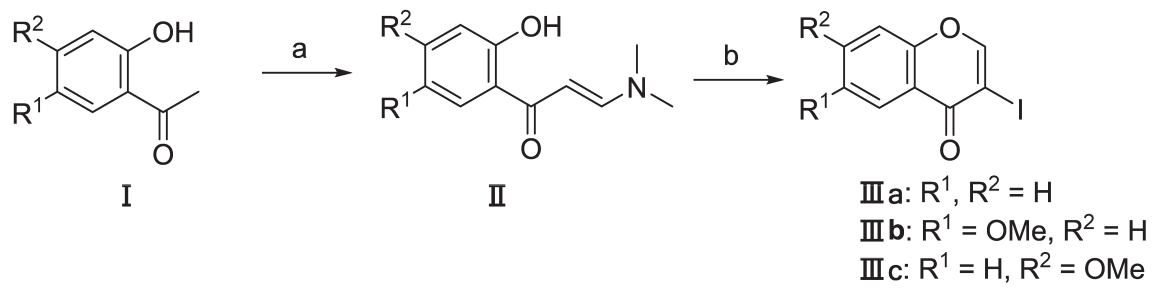<smiles>[R]c1cc2occ(I)c(=O)c2cc1[R]</smiles>

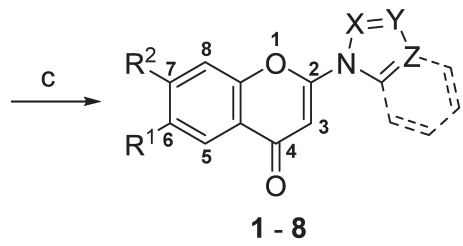

$\mathrm{X}, \mathrm{Y}, \mathrm{Z}=\mathrm{N}, \mathrm{CH}$ or $\mathrm{C}$<smiles>[R]c1cc2oc(-n3cccc3)cc(=O)c2cc1[R]</smiles>

1a: $R^{1}, R^{2}=H$ 1b: $R^{1}=O M e, R^{2}=H$ 1c: $R^{1}=H, R^{2}=O M e$<smiles>[R]c1cc2oc(-n3cccn3)cc(=O)c2cc1[R]</smiles>

2a: $R^{1}, R^{2}=H$ 2b: $R^{1}=O M e, R^{2}=H$ 2c: $R^{1}=H, R^{2}=O M e$<smiles>[R]c1cc2oc(-n3ccnc3)cc(=O)c2cc1[R]</smiles>

3a: $R^{1}, R^{2}=H$

3b: $R^{1}=O M e, R^{2}=H$ 3c: $R^{1}=H, R^{2}=O M e$<smiles>[R]c1cc2oc(-n3cncn3)cc(=O)c2cc1[R]</smiles>

4a: $R^{1}, R^{2}=H$

4b: $\mathrm{R}^{1}=\mathrm{OMe}, \mathrm{R}^{2}=\mathrm{H}$

4c: $R^{1}=H, R^{2}=O M e$<smiles>[R]c1cc2oc(-n3ccnn3)cc(=O)c2cc1[R]</smiles>

5a: $R^{1}, R^{2}=H$ 5b: $R^{1}=O M e, R^{2}=H$ 5c: $R^{1}=H, R^{2}=O M e$<smiles>[R]c1cc2oc(-n3ccc4ccccc43)cc(=O)c2cc1[R]</smiles>

6a: $R^{1}, R^{2}=H$ 6b: $\mathrm{R}^{1}=\mathrm{OMe}, \mathrm{R}^{2}=\mathrm{H}$ 6c: $R^{1}=H, R^{2}=O M e$<smiles>[R]c1cc2oc(-n3ncc4ccccc43)cc(=O)c2cc1[R]</smiles>

7a: $R^{1}, R^{2}=H$ 7b: $R^{1}=O M e, R^{2}=H$ 7c: $\mathrm{R}^{1}=\mathrm{H}, \mathrm{R}^{2}=\mathrm{OMe}$<smiles>[R]c1cc2oc(-n3cnc4ccccc43)cc(=O)c2cc1[R]</smiles>

8a: $R^{1}, R^{2}=H$

8b: $R^{1}=O M e, R^{2}=H$

8c: $R^{1}=H, R^{2}=O M e$

Reagents and conditions: (a) DMF-DMA, $95^{\circ} \mathrm{C}$; (b) Pyridine, $\mathrm{I}_{2}, \mathrm{CHCl}_{3}$; (c) Azole, $\mathrm{K}_{2} \mathrm{CO}_{3}, \mathrm{DMF}, 80^{\circ} \mathrm{C}$.

Chart 1. Synthetic Protocol for the 2-Azolylchromone Derivatives

rivatives.

The MAO-B inhibitory activities of the 2-azolylchromone derivatives were also determined, with 6 of the 24 tested 2-azolylchromones displaying various degrees of potency. Compound $4 \mathbf{c}$ was the most potent inhibitor $\left(\mathrm{IC}_{50}=0.019 \mu \mathrm{M}\right)$. Compounds 4a, 5a, c, 6c and 8c showed potent inhibitory activities, with sub-micro molar $\mathrm{IC}_{50}$ values. 7-Methoxy substituted compounds, such as $\mathbf{4 c}, \mathbf{5 c}, \mathbf{6 c}$ and $\mathbf{8 c}$, showed potent inhibitory activities against MAO-B, and compounds $\mathbf{1 c}$, $\mathbf{2 c}$, $3 \mathbf{c}$ and $7 \mathbf{c}$ also showed selectivity for MAO-B compared with the unsubstituted or 6-methoxy substituted 2-azolylchromone derivatives. These results suggested that introduction of a methoxy group on the chromone ring of 2-azolylchromone derivatives induced selectivities for MAO-A and MAO-B (Fig. 1).

In addition, compounds $\mathbf{1 b}, \mathbf{2 b}$ and $\mathbf{7 b}$ showed higher selectivity for MAO-A, and compounds $\mathbf{6 c}$ and $\mathbf{8 c}$ showed higher selectivity for MAO-B. These differences might be due to the size of the active site cavity. The active site structures of MAO-A and MAO-B have been reported and the substrate cavity in MAO-A is smaller than the cavity in MAO-B. ${ }^{3-5)}$

Interestingly, compounds $\mathbf{4 a - c}$ and $\mathbf{5 a}-\mathbf{c}$ showed potent inhibitory activities against both MAO-A and MAO-B, sug- gesting that the triazole moiety is recognized by MAOs. Thus, the 2-triazolylchromone structure might be a useful scaffold for the design of MAO inhibitors.

This is the first report identifying 2-azolylchromone derivatives as potent MAO inhibitors. The biological activity of 2-azolylchrmone was only recently reported and is a potent hedgehog signaling pathway inhibitor. ${ }^{19)}$

\section{Conclusion}

We synthesized a series of 2-azolylchromone derivatives and evaluated their potential as potent and selective MAO-A and MAO-B inhibitors. Compounds $\mathbf{1 b}, \mathbf{2 b}, \mathbf{4} \mathbf{a}-\mathbf{c}, \mathbf{5 b}$ and 7b showed potent inhibitory activities against MAO-A, and compounds $4 \mathbf{a}, \mathbf{c}, \mathbf{5 a}, \mathbf{c}, \mathbf{6 c}$ and $\mathbf{8 c}$ showed potent inhibitory activities against MAO-B. These data suggest that 6-methoxy substitution of 2-azolylchromones favors MAO-A inhibition and that 7-methoxy substitution favors MAO-B inhibition. Compound $\mathbf{4 b}$ was the most potent inhibitor $\left(\mathrm{IC}_{50}=0.023 \mu \mathrm{M}\right)$ for MAO-A, and compound $\mathbf{4 c}$ was the most potent inhibitor $\left(\mathrm{IC}_{50}=0.019 \mu \mathrm{M}\right)$ for MAO-B. This is the first report identifying 2-azolylchromone derivatives as potent MAO inhibitors. These results suggest that the 2-triazolylchromone structure 
Table 1. The $\mathrm{IC}_{50}$ Values and Selectivity Indices for MAO-A and MAO-B of the 2-Azolylchromone Derivatives

\begin{tabular}{|c|c|c|c|c|c|c|}
\hline \multirow{2}{*}{ Compd. } & \multirow{2}{*}{$\mathrm{R}^{1}$} & \multirow{2}{*}{$\mathrm{R}^{2}$} & \multicolumn{2}{|c|}{$\mathrm{IC}_{50}(\mu \mathrm{M})$} & \multicolumn{2}{|c|}{ Selectivity index } \\
\hline & & & MAO-A & MAO-B & For MAO-A & For MAO-B \\
\hline $1 \mathrm{a}$ & $\mathrm{H}$ & $\mathrm{H}$ & 4.56 & $>100$ & $>20$ & - \\
\hline $1 \mathrm{~b}$ & $\mathrm{MeO}$ & $\mathrm{H}$ & 0.32 & $>100$ & $>300$ & - \\
\hline 1c & $\mathrm{H}$ & $\mathrm{MeO}$ & 14.12 & 7.93 & - & - \\
\hline $2 a$ & $\mathrm{H}$ & $\mathrm{H}$ & 1.83 & 2.87 & - & - \\
\hline $2 \mathrm{~b}$ & $\mathrm{MeO}$ & $\mathrm{H}$ & 0.14 & $>100$ & $>700$ & - \\
\hline $2 \mathrm{c}$ & $\mathrm{H}$ & $\mathrm{MeO}$ & 2.07 & 1.04 & - & - \\
\hline $3 \mathbf{a}$ & $\mathrm{H}$ & $\mathrm{H}$ & 25.36 & 6.11 & - & 4 \\
\hline $3 b$ & $\mathrm{MeO}$ & $\mathrm{H}$ & 6.73 & 19.5 & 3 & - \\
\hline $3 c$ & $\mathrm{H}$ & $\mathrm{MeO}$ & 26.25 & 5.29 & 一 & 5 \\
\hline $4 a$ & $\mathrm{H}$ & $\mathrm{H}$ & 0.11 & 0.028 & - & 4 \\
\hline $4 b$ & $\mathrm{MeO}$ & $\mathrm{H}$ & 0.023 & 1.64 & 80 & - \\
\hline $4 c$ & $\mathrm{H}$ & $\mathrm{MeO}$ & 0.15 & 0.019 & - & 8 \\
\hline $5 a$ & $\mathrm{H}$ & $\mathrm{H}$ & 4.43 & 0.73 & - & 6 \\
\hline $5 b$ & $\mathrm{MeO}$ & $\mathrm{H}$ & 0.59 & 15.65 & 30 & - \\
\hline $5 c$ & $\mathrm{H}$ & $\mathrm{MeO}$ & 6.18 & 0.28 & - & 20 \\
\hline $6 a$ & $\mathrm{H}$ & $\mathrm{H}$ & 6.19 & 2.56 & - & - \\
\hline $6 b$ & $\mathrm{MeO}$ & $\mathrm{H}$ & 1.00 & 2.01 & - & - \\
\hline $6 c$ & $\mathrm{H}$ & $\mathrm{MeO}$ & $>100$ & 0.28 & - & $>300$ \\
\hline $7 a$ & $\mathrm{H}$ & $\mathrm{H}$ & 1.58 & $>100$ & $>60$ & - \\
\hline $7 b$ & $\mathrm{MeO}$ & $\mathrm{H}$ & 0.19 & $>100$ & $>500$ & - \\
\hline $7 c$ & $\mathrm{H}$ & $\mathrm{MeO}$ & 34.37 & 25.07 & - & - \\
\hline $8 a$ & $\mathrm{H}$ & $\mathrm{H}$ & $>100$ & $>100$ & - & - \\
\hline $8 b$ & $\mathrm{MeO}$ & $\mathrm{H}$ & 3.41 & 2.73 & - & - \\
\hline $8 c$ & $\mathrm{H}$ & $\mathrm{MeO}$ & $>100$ & 0.27 & - & $>300$ \\
\hline Pargyline & - & - & 4.57 & 0.22 & - & 20 \\
\hline
\end{tabular}

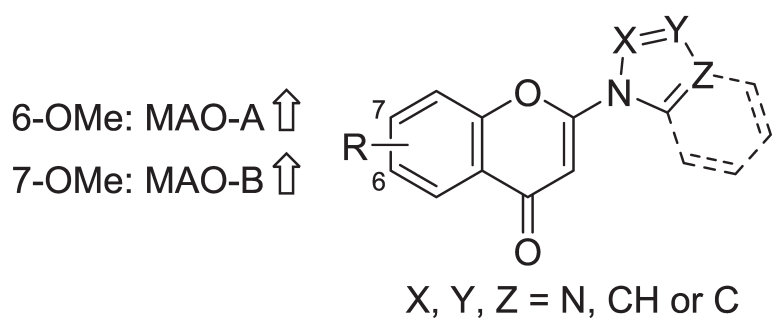

Fig. 1. Methoxy Substitution on the Chromone Ring Induced Selectivity for MAO-A and MAO-B

might be a useful scaffold for the design and development of novel MAO inhibitors.

\section{Experimental}

Chemistry All reagents and solvents were purchased from commercial sources. Analytical TLC was performed on silicacoated plates (silica gel 60F-254, Merck) and compounds were visualized under UV light. Column chromatography was carried out using silica gel (Wakogel C-200, Wako). All melting points were determined using a Yanagimoto micro-hot stage and are uncorrected. ${ }^{1} \mathrm{H}-\mathrm{NMR}$ and ${ }^{13} \mathrm{C}-\mathrm{NMR}$ spectra were recorded on a Varian $400-\mathrm{MR} 400 \mathrm{MHz}$ spectrometer using tetramethylsilane as the internal standard. MS spectra were measured using a JEOL JMS-700 spectrometer. Elemental analyses were carried out on a Yanaco CHN MT-6 elemental analyzer.

Synthesis of 3-Iodochromone Derivatives 3-Iodochromone derivatives (IIIa-c) were synthesized according to a previously reported method. ${ }^{18)}$ The products (IIIa-c) were identified from their melting points and ${ }^{1} \mathrm{H}-\mathrm{NMR}$ spectra.
3-Iodo-4H-1-benzopyran-4-one (IIIa) Pale yellow prism. mp $97-98^{\circ} \mathrm{C} .{ }^{1} \mathrm{H}-\mathrm{NMR}\left(\mathrm{CDCl}_{3}, 400 \mathrm{MHz}\right) \delta$ : $7.48(1 \mathrm{H}, \mathrm{dd}$, $J=8.5,1.1 \mathrm{~Hz}, \mathrm{H}-8), 7.46(1 \mathrm{H}$, ddd, $J=8.0,7.1,1.1 \mathrm{~Hz}, \mathrm{H}-6)$, $7.72(1 \mathrm{H}$, ddd, $J=8.5,7.1,1.7 \mathrm{~Hz}, \mathrm{H}-7), 7.48(1 \mathrm{H}, \mathrm{dd}, J=8.0$, $1.7 \mathrm{~Hz}, \mathrm{H}-5), 8.31$ (1H, s, H-2). MS-electron ionization (EI) $m / z: 272\left[\mathrm{M}^{+}\right]$.

The ${ }^{1} \mathrm{H}-\mathrm{NMR}$ spectrum was similar to that previously reported. ${ }^{18)}$

3-Iodo-6-methoxy-4H-1-benzopyran-4-one (IIIb) White needle crystal. mp $114-115^{\circ} \mathrm{C} .{ }^{1} \mathrm{H}-\mathrm{NMR}\left(\mathrm{CDCl}_{3}, 400 \mathrm{MHz}\right) \delta$ : $3.91(3 \mathrm{H}, \mathrm{s}, \mathrm{OMe}), 7.30(1 \mathrm{H}, \mathrm{dd}, J=9.1,3.1 \mathrm{~Hz}, \mathrm{H}-7), 7.42(1 \mathrm{H}$, d, $J=9.0 \mathrm{~Hz}, \mathrm{H}-8), 7.59(1 \mathrm{H}, \mathrm{d}, J=3.1 \mathrm{~Hz}, \mathrm{H}-5), 8.29(1 \mathrm{H}, \mathrm{s}$, H-2). MS-EI $m / z: 302\left[\mathrm{M}^{+}\right]$.

The ${ }^{1} \mathrm{H}-\mathrm{NMR}$ spectrum was similar to that previously reported. ${ }^{18)}$

3-Iodo-7-methoxy-4H-1-benzopyran-4-one (IIIc) Colorless prism. mp $165-166^{\circ} \mathrm{C} .{ }^{1} \mathrm{H}-\mathrm{NMR}\left(\mathrm{CDCl}_{3}, 400 \mathrm{MHz}\right) \delta$ : $3.91(3 \mathrm{H}, \mathrm{s}, \mathrm{OMe}), 6.84(1 \mathrm{H}, \mathrm{d}, J=2.4 \mathrm{~Hz}, \mathrm{H}-8), 7.00(1 \mathrm{H}, \mathrm{dd}$, $J=9.0,2.4 \mathrm{~Hz}, \mathrm{H}-6), 8.15(1 \mathrm{H}, \mathrm{d}, J=9.0 \mathrm{~Hz}, \mathrm{H}-5), 8.22(1 \mathrm{H}, \mathrm{s}$, H-2). MS-EI $m / z: 302\left[\mathrm{M}^{+}\right]$.

The ${ }^{1} \mathrm{H}-\mathrm{NMR}$ spectrum was similar to that previously reported. ${ }^{18)}$

General Procedure for Preparation of 2-Azolylchromone Derivatives 2-Azolylchromone derivatives were synthesized according to a previously reported method. ${ }^{17)} \mathrm{A}$ mixture of 3-iodochromone (1 mmol), azole $(2 \mathrm{mmol})$ and $\mathrm{K}_{2} \mathrm{CO}_{3}$ (10 mmol) in $N, N$-dimethylformamide (DMF) $(5 \mathrm{~mL})$ was stirred at $80^{\circ} \mathrm{C}$ for $24 \mathrm{~h}$. The reaction mixture was extracted with water and $\mathrm{CHCl}_{3}$. The organic layer was dried over $\mathrm{Na}_{2} \mathrm{SO}_{4}$ and the solvent was evaporated in vacuo. The residue was purified by silica gel column chromatography (hexane- 
ethyl acetate) to give 2-azolylchromone derivatives.

2-(1H-Pyrrol-1-yl)-4H-1-benzopyran-4-one (1a) Yield $63 \%$. Brown prism. mp $134-136^{\circ} \mathrm{C}$. IR $(\mathrm{KBr}) \mathrm{cm}^{-1}$ : 586, 725, 779, 1056, 1388, 1419, 1566, 1635, 3070, 3116. ${ }^{1} \mathrm{H}-\mathrm{NMR}$ $\left(\mathrm{CDCl}_{3}, 400 \mathrm{MHz}\right) \delta: 6.32(1 \mathrm{H}, \mathrm{s}, \mathrm{H}-3), 6.45(2 \mathrm{H}, \mathrm{t}, J=2.3 \mathrm{~Hz}$, H-3', 4'), 7.33 (2H, t, $\left.J=2.3 \mathrm{~Hz}, \mathrm{H}-2^{\prime}, 5^{\prime}\right), 7.46$ (1H, ddd, $J=7.9$, $7.2,1.0 \mathrm{~Hz}, \mathrm{H}-6), 7.52(1 \mathrm{H}, \mathrm{dd}, J=8.4,1.0 \mathrm{~Hz}, \mathrm{H}-8), 7.72(1 \mathrm{H}$, ddd, $J=8.4,7.2,1.7 \mathrm{~Hz}, \mathrm{H}-7), 8.23(1 \mathrm{H}, \mathrm{dd}, J=7.9,1.7 \mathrm{~Hz}, \mathrm{H}-5)$. ${ }^{13} \mathrm{C}-\mathrm{NMR}\left(\mathrm{CDCl}_{3}, 100 \mathrm{MHz}\right) \delta: 94.8,113.4,117.4,118.2,123.4$, 125.7, 125.9, 133.7, 154.1, 155.7, 178.1. MS-EI m/z: $211\left[\mathrm{M}^{+}\right]$.

The ${ }^{1} \mathrm{H}-\mathrm{NMR}$ spectrum was similar to that previously reported. ${ }^{17)}$

6-Methoxy-2-(1H-pyrrol-1-yl)-4H-1-benzopyran-4-one (1b) Yield 47\%. Pale brown solid. mp $146-147^{\circ} \mathrm{C}$. IR (KBr) $\mathrm{cm}^{-1}$ : 555, 748, 1265, 1381, 1435, 1581, 1643, 3070, 3116. ${ }^{1} \mathrm{H}-\mathrm{NMR}\left(\mathrm{CDCl}_{3}, 400 \mathrm{MHz}\right) \delta: 3.90(3 \mathrm{H}, \mathrm{s}, \mathrm{OMe}), 6.32(1 \mathrm{H}$, s, H-3), $6.44\left(2 \mathrm{H}, \mathrm{t} J=2.3 \mathrm{~Hz}, \mathrm{H}-3^{\prime}, 4^{\prime}\right), 7.27(1 \mathrm{H}, \mathrm{dd}, J=9.1$, $3.1 \mathrm{~Hz}, \mathrm{H}-7), 7.32\left(2 \mathrm{H}, \mathrm{t}, J=2.3 \mathrm{~Hz}, \mathrm{H}-2^{\prime}, 5^{\prime}\right), 7.45(1 \mathrm{H}, \mathrm{d}$, $J=9.1 \mathrm{~Hz}, \mathrm{H}-8), 7.60(1 \mathrm{H}, \mathrm{d}, J=3.1 \mathrm{~Hz}, \mathrm{H}-5) \cdot{ }^{13} \mathrm{C}-\mathrm{NMR}\left(\mathrm{CDCl}_{3}\right.$, $100 \mathrm{MHz}) \delta: 60.0,94.3,105.4,113.4,118.2,118.7,123.3,124.0$, 148.8, 155.6, 157.3, 178.0. MS-EI m/z: $241\left[\mathrm{M}^{+}\right]$. Anal. Calcd for $\mathrm{C}_{14} \mathrm{H}_{11} \mathrm{NO}_{3}$ : C, 69.70; $\mathrm{H}, 4.60 ; \mathrm{N}, 5.81$. Found: C, 69.42; H, 4.55; N, 5.62 .

7-Methoxy-2-(1H-pyrrol-1-yl)-4H-1-benzopyran-4-one (1c) Yield $57 \%$. Pale brown prism. mp $148-150^{\circ} \mathrm{C}$. IR ( $\left.\mathrm{KBr}\right)$ $\mathrm{cm}^{-1}$ : 547, 740, 856, 1172, 1280, 1388, 1604, 1651, 3070, 3132. ${ }^{1} \mathrm{H}-\mathrm{NMR}\left(\mathrm{CDCl}_{3}, 400 \mathrm{MHz}\right) \delta: 3.94(3 \mathrm{H}, \mathrm{s}, \mathrm{OMe}), 6.25(1 \mathrm{H}, \mathrm{s}$, $\mathrm{H}-3), 6.44$ (2H, t, $\left.J=2.3 \mathrm{~Hz}, \mathrm{H}-3^{\prime}, 4^{\prime}\right), 6.93(1 \mathrm{H}, \mathrm{d}, J=2.4 \mathrm{~Hz}$, $\mathrm{H}-8), 7.02(1 \mathrm{H}, \mathrm{dd}, J=8.8,2.4 \mathrm{~Hz}, \mathrm{H}-6), 7.31(2 \mathrm{H}, \mathrm{t}, J=2.3 \mathrm{~Hz}$ $\left.\mathrm{H}-2^{\prime}, 5^{\prime}\right), 8.13(1 \mathrm{H}, \mathrm{d}, J=8.8 \mathrm{~Hz}, \mathrm{H}-5) .{ }^{13} \mathrm{C}-\mathrm{NMR}\left(\mathrm{CDCl}_{3}\right.$, $100 \mathrm{MHz}) \delta: 55.9,94.6,100.4,113.2,114.3,117.1,118.2,127.2$, 155.5, 155.8, 164.1, 177.7. MS-EI m/z: $241\left[\mathrm{M}^{+}\right]$. Anal. Calcd for $\mathrm{C}_{14} \mathrm{H}_{11} \mathrm{NO}_{3}$ : C, 69.70; $\mathrm{H}, 4.60 ; \mathrm{N}$, 5.81. Found: C, 69.62; H, 4.62; N, 5.71.

2-(1H-Pyrazol-1-yl)-4H-1-benzopyran-4-one (2a) Yield $70 \%$. White solid. mp $142-143^{\circ} \mathrm{C}$. IR (KBr) cm ${ }^{-1}$ : 948, 1222, 1396, 1417, 1573, 1635, 3039, 3103. ${ }^{1} \mathrm{H}-\mathrm{NMR}\left(\mathrm{CDCl}_{3}\right.$, $400 \mathrm{MHz}) \delta: 6.60\left(1 \mathrm{H}, \mathrm{dd}, J=2.7,1.6 \mathrm{~Hz}, \mathrm{H}-4^{\prime}\right), 6.91(1 \mathrm{H}, \mathrm{s}$, $\mathrm{H}-3), 7.47$ (1H, ddd, J=7.9, 7.1, $1.0 \mathrm{~Hz}, \mathrm{H}-6), 7.55(1 \mathrm{H}, \mathrm{dd}$, $J=8.5,1.0 \mathrm{~Hz}, \mathrm{H}-8), 7.72(1 \mathrm{H}, \mathrm{ddd}, J=8.5,7.1,1.7 \mathrm{~Hz}, \mathrm{H}-7)$, $7.86\left(1 \mathrm{H}, \mathrm{d}, J=1.6 \mathrm{~Hz}, \mathrm{H}-3^{\prime}\right), 8.24\left(1 \mathrm{H}, \mathrm{d}, J=2.7 \mathrm{~Hz}, \mathrm{H}-5^{\prime}\right)$, $8.26(1 \mathrm{H}, \mathrm{dd}, J=7.9,1.7 \mathrm{~Hz}, \mathrm{H}-5) .{ }^{13} \mathrm{C}-\mathrm{NMR}\left(\mathrm{CDCl}_{3}, 100 \mathrm{MHz}\right)$ $\delta$ : 96.3, 109.6, 117.4, 123.7, 125.9, 126.1, 127.7, 133.8, 144.3, 154.0, 155.2, 177.9. MS-EI $m / z: 212\left[\mathrm{M}^{+}\right]$.

The ${ }^{1} \mathrm{H}-\mathrm{NMR}$ spectrum was similar to that previously reported. ${ }^{17)}$

6-Methoxy-2-(1 H-pyrazol-1-yl)-4H-1-benzopyran-4-one (2b) Yield $67 \%$. Pale yellow needle crystal. mp $172-173^{\circ} \mathrm{C}$. IR (KBr) $\mathrm{cm}^{-1}$ : 555, 825, 887, 1018, 1211, 1396, 1581, 1635, 3039, 3101. ${ }^{1} \mathrm{H}-\mathrm{NMR}\left(\mathrm{CDCl}_{3}, 400 \mathrm{MHz}\right) \delta: 3.92(3 \mathrm{H}, \mathrm{s}$, OMe), 6.58 (1H, m, H-4'), 6.90 (1H, s, H-3), $7.29(1 \mathrm{H}, \mathrm{dd}$, $J=9.1,3.1 \mathrm{~Hz}, \mathrm{H}-7), 7.47$ (1H, d, $J=9.1 \mathrm{~Hz}, \mathrm{H}-8), 7.63(1 \mathrm{H}$, d, $J=3.1 \mathrm{~Hz}, \mathrm{H}-5), 7.85\left(1 \mathrm{H}, \mathrm{d}, J=1.6 \mathrm{~Hz}, \mathrm{H}-3^{\prime}\right), 8.22(1 \mathrm{H}$, d, $\left.J=2.7 \mathrm{~Hz}, \mathrm{H}-5^{\prime}\right) .{ }^{13} \mathrm{C}-\mathrm{NMR}\left(\mathrm{CDCl}_{3}, 100 \mathrm{MHz}\right) \delta: 56.0$, 95.8, 105.6, 109.5, 118.8, 123.5, 124.4, 127.7, 144.2, 148.6, 155.2, 157.4, 177.9. MS-EI m/z: $242\left[\mathrm{M}^{+}\right]$. Anal. Calcd for $\mathrm{C}_{13} \mathrm{H}_{10} \mathrm{~N}_{2} \mathrm{O}_{3}: \mathrm{C}, 64.46 ; \mathrm{H}, 4.16 ; \mathrm{N}, 11.56$. Found: $\mathrm{C}$ 64.24; H, 4.05; N, 11.43.

7-Methoxy-2-(1H-pyrazol-1-yl)-4H-1-benzopyran-4-one (2c) Yield $89 \%$. Pale yellow needle crystal. mp $173^{\circ} \mathrm{C}$. IR (KBr) $\mathrm{cm}^{-1}$ : 594, 763, 817, 1257, 1404, 1635, 3039, 3109.
${ }^{1} \mathrm{H}-\mathrm{NMR} \quad\left(\mathrm{CDCl}_{3}, 400 \mathrm{MHz}\right) \quad \delta: 3.94(3 \mathrm{H}, \quad \mathrm{s}, \mathrm{OMe}), 6.58$ $\left(1 \mathrm{H}, \mathrm{dd}, J=2.7,1.6 \mathrm{~Hz}, \mathrm{H}-4^{\prime}\right), 6.82(1 \mathrm{H}, \mathrm{s}, \mathrm{H}-3), 6.95(1 \mathrm{H}$, d, $J=2.3 \mathrm{~Hz}, \mathrm{H}-8), 7.02(1 \mathrm{H}, \mathrm{dd}, J=8.8,2.4 \mathrm{~Hz}, \mathrm{H}-6), 7.84$ $\left(1 \mathrm{H}, \mathrm{d}, J=1.6 \mathrm{~Hz}, \mathrm{H}-3^{\prime}\right), 8.15(1 \mathrm{H}, \mathrm{d}, J=8.8 \mathrm{~Hz}, \mathrm{H}-5), 8.20$ $\left(1 \mathrm{H}, \mathrm{d}, J=2.7 \mathrm{~Hz}, \mathrm{H}-5^{\prime}\right) .{ }^{13} \mathrm{C}-\mathrm{NMR}\left(\mathrm{CDCl}_{3}, 100 \mathrm{MHz}\right) \delta: 55.9$, 96.2, 100.4, 109.4, 114.4, 117.4, 127.3, 127.6, 144.1, 155.0, 155.7, 164.1, 177.5. MS-EI m/z: $242\left[\mathrm{M}^{+}\right]$. Anal. Calcd for $\mathrm{C}_{13} \mathrm{H}_{10} \mathrm{~N}_{2} \mathrm{O}_{3}$ : C, 64.46; H, 4.16; N, 11.56. Found: C, 64.46; H, $4.12 ; \mathrm{N}, 11.54$.

2-(1H-Imidazol-1-yl)-4H-1-benzopyran-4-one (3a) Yield $81 \%$. White solid. mp $197-198^{\circ} \mathrm{C}$. IR $(\mathrm{KBr}) \mathrm{cm}^{-1}: 617,756$, $848,887,1056,1265,1303,1427,1635,3062,3116 .{ }^{1} \mathrm{H}-\mathrm{NMR}$ $\left(\mathrm{CDCl}_{3}, 400 \mathrm{MHz}\right) \delta: 6.38(1 \mathrm{H}, \mathrm{s}, \mathrm{H}-3), 7.27-7.28(1 \mathrm{H}, \mathrm{m}$, H-4'), 7.46-7.48 (1H, m, H-5'), $7.49(1 \mathrm{H}, \mathrm{ddd}, J=7.9,7.2$, $1.0 \mathrm{~Hz}, \mathrm{H}-6), 7.54$ (1H, dd, $J=8.4,1.0 \mathrm{~Hz}, \mathrm{H}-8), 7.75$ (1H, ddd, $J=8.4,7.2,1.7 \mathrm{~Hz}, \mathrm{H}-7), 8.20-8.23\left(1 \mathrm{H}, \mathrm{m}, \mathrm{H}-2^{\prime}\right), 8.23(1 \mathrm{H}$, $\mathrm{dd}, J=7.2,1.7 \mathrm{~Hz}, \mathrm{H}-5) .{ }^{13} \mathrm{C}-\mathrm{NMR}\left(\mathrm{CDCl}_{3}, 100 \mathrm{MHz}\right) \delta: 97.1$, $115.8,117.6,123.4,126.0,126.2,131.9,134.3,134.8,153.3$, 154.2, 177.7. MS-EI $m / z: 212\left[\mathrm{M}^{+}\right]$.

The ${ }^{1} \mathrm{H}-\mathrm{NMR}$ spectrum was similar to that previously reported. ${ }^{17)}$

2-(1H-Imidazol-1-yl)-6-methoxy-4H-1-benzopyran-4-one (3b) Yield $72 \%$. White solid. mp $199^{\circ} \mathrm{C}$. IR $(\mathrm{KBr}) \mathrm{cm}^{-1}: 825$, 1249, 1303, 1458, 1643, 3062. ${ }^{1} \mathrm{H}-\mathrm{NMR}\left(\mathrm{CDCl}_{3}, 400 \mathrm{MHz}\right) \delta$ : $3.92(3 \mathrm{H}, \mathrm{s}, \mathrm{OMe}), 6.37$ (1H, s, H-3), 7.25-7.27 (1H, m, H-4'), $7.32(1 \mathrm{H}, \mathrm{dd}, J=9.1,3.1 \mathrm{~Hz}, \mathrm{H}-7), 7.45-7.47$ (1H, m, H-5'), 7.48 $(1 \mathrm{H}, \mathrm{d}, J=9.1 \mathrm{~Hz}, \mathrm{H}-8), 7.59(1 \mathrm{H}, \mathrm{d}, J=3.1 \mathrm{~Hz}, \mathrm{H}-5), 8.19-8.21$ $\left(1 \mathrm{H}, \mathrm{m}, \mathrm{H}-2^{\prime}\right) .{ }^{13} \mathrm{C}-\mathrm{NMR}\left(\mathrm{CDCl}_{3}, 100 \mathrm{MHz}\right) \delta$ : 56.0, 96.5, $105.5,115.8,119.0,124.0,124.1,131.8,134.7,148.8,153.2$, 157.7, 177.7. MS-EI $m / z: 242\left[\mathrm{M}^{+}\right]$. Anal. Calcd for $\mathrm{C}_{13} \mathrm{H}_{10} \mathrm{~N}_{2} \mathrm{O}_{3}$ : C, 64.46; H, 4.16; N, 11.56. Found: C, 64.21; H, 4.20; N, 11.56.

2-(1H-Imidazol-1-yl)-7-methoxy-4H-1-benzopyran-4-one (3c) Yield $89 \%$. White solid. mp $177-179^{\circ} \mathrm{C}$. IR $(\mathrm{KBr}) \mathrm{cm}^{-1}$ : 455, 648, 810, 1002, 1049, 1087, 1242, 1296, 1427, 1643, 3062. ${ }^{1} \mathrm{H}-\mathrm{NMR}\left(\mathrm{CDCl}_{3}, 400 \mathrm{MHz}\right) \delta: 3.94(3 \mathrm{H}, \mathrm{s}, \mathrm{OMe}), 6.31(1 \mathrm{H}, \mathrm{s}$, $\mathrm{H}-3), 6.94(1 \mathrm{H}, \mathrm{d}, J=2.4 \mathrm{~Hz}, \mathrm{H}-8), 7.04(1 \mathrm{H}, \mathrm{dd}, J=8.9,2.4 \mathrm{~Hz}$, H-6), 7.25-7.26 (1H, m, H-4' $), 7.43-7.44$ (1H, m, H-5'), 8.12 $(1 \mathrm{H}, \mathrm{d}, J=8.9 \mathrm{~Hz}, \mathrm{H}-5), 8.19-8.20\left(1 \mathrm{H}, \mathrm{m}, \mathrm{H}-2^{\prime}\right) .{ }^{13} \mathrm{C}-\mathrm{NMR}$ $\left(\mathrm{CDCl}_{3}, 100 \mathrm{MHz}\right) \delta: 56.0,97.0,100.4,114.9,115.8,117.0$, 127.3, 131.7, 134.7, 153.0, 155.9, 164.5, 177.2. MS-EI $m / z: 242$ $\left[\mathrm{M}^{+}\right]$. Anal. Calcd for $\mathrm{C}_{13} \mathrm{H}_{10} \mathrm{~N}_{2} \mathrm{O}_{3}: \mathrm{C}, 64.46 ; \mathrm{H}, 4.16, \mathrm{~N} ; 11.56$. Found: C, 63.20; H, 4.14; N, 11.56 .

2-(1H-1,2,4-Triazol-1-yl)-4H-1-benzopyran-4-one (4a) Yield $73 \%$. White solid. mp $205-207^{\circ} \mathrm{C}$. IR (KBr) $\mathrm{cm}^{-1}: 609$, 655, 748, 902, 987, 1064, 1234, 1427, 1512, 1635, 3008, 3101. ${ }^{1} \mathrm{H}-\mathrm{NMR}\left(\mathrm{CDCl}_{3}, 400 \mathrm{MHz}\right) \delta: 6.91(1 \mathrm{H}, \mathrm{s}, \mathrm{H}-3), 7.52(1 \mathrm{H}$, ddd, $J=7.9,7.2,1.0 \mathrm{~Hz}, \mathrm{H}-6), 7.58(1 \mathrm{H}, \mathrm{dd}, J=8.5,1.0 \mathrm{~Hz}$, $\mathrm{H}-8), 7.78(1 \mathrm{H}$, ddd, $J=8.5,7.2,1.7 \mathrm{~Hz}, \mathrm{H}-7), 8.19$ (1H, s, H-3'), 8.27 (1H, dd, J=7.9, $1.7 \mathrm{~Hz}, \mathrm{H}-5), 8.92$ (1H, s, H-5'). ${ }^{13} \mathrm{C}-\mathrm{NMR}\left(\mathrm{CDCl}_{3}, 100 \mathrm{MHz}\right) \delta: 98.2,117.5,123.7,126.3,126.4$, 134.4, 142.0, 152.9, 153.9, 154.0, 177.6. MS-EI m/z: $213\left[\mathrm{M}^{+}\right]$.

The ${ }^{1} \mathrm{H}-\mathrm{NMR}$ spectrum was similar to that previously reported. ${ }^{17)}$

6-Methoxy-2-(1H-1,2,4-triazol-1-yl)-4H-1-benzopyran4-one (4b) Yield 61\%. Pale yellow solid. mp $233-235^{\circ} \mathrm{C}$. IR $(\mathrm{KBr}) \mathrm{cm}^{-1}:$ 663, 825, 902, 987, 1026, 1234, 1280, 1357, 1419, 1635, 3109. ${ }^{1} \mathrm{H}-\mathrm{NMR}\left(\mathrm{CDCl}_{3}, 400 \mathrm{MHz}\right) \delta: 3.96$ (3H, s, OMe), 6.89 (1H, s, H-3), 7.34 (1H, dd, $J=9.1,3.1 \mathrm{~Hz}, \mathrm{H}-7), 7.50(1 \mathrm{H}$, d, $J=9.1 \mathrm{~Hz}, \mathrm{H}-8), 7.63(1 \mathrm{H}, \mathrm{d}, J=3.1 \mathrm{~Hz}, \mathrm{H}-5), 8.18(1 \mathrm{H}, \mathrm{s}$, $\left.\mathrm{H}-3^{\prime}\right), 8.90\left(1 \mathrm{H}, \mathrm{s}, \mathrm{H}-5^{\prime}\right) .{ }^{13} \mathrm{C}-\mathrm{NMR}\left(\mathrm{CDCl}_{3}, 100 \mathrm{MHz}\right) \delta: 56.0$, 97.6, 105.7, 118.9, 124.1, 124.4, 141.9, 148.6, 152.8, 153.9, 157.6, 
177.6. MS-EI $m / z: 243\left[\mathrm{M}^{+}\right]$. Anal. Calcd for $\mathrm{C}_{12} \mathrm{H}_{9} \mathrm{~N}_{3} \mathrm{O}_{3}: \mathrm{C}$, 59.26; H, 3.73; N, 17.28. Found: C, 59.15; H, 3.59; N, 17.14.

7-Methoxy-2-(1H-1,2,4-triazol-1-yl)-4H-1-benzopyran4-one (4c) Yield 82\%. Yellow needle crystal. mp $233-235^{\circ} \mathrm{C}$. IR (KBr) $\mathrm{cm}^{-1}$ : 825, 902, 995, 1080, 1126, 1234, 1411, 1512, 1612, 3008, 3086. ${ }^{1} \mathrm{H}-\mathrm{NMR}\left(\mathrm{CDCl}_{3}, 100 \mathrm{MHz}\right) \delta$ : $3.96(3 \mathrm{H}, \mathrm{s}$, OMe), 6.84 (1H, s, H-3), 6.97 (1H, d, J=2.3 Hz, H-8), $7.06(1 \mathrm{H}$, dd, $J=8.9,2.3 \mathrm{~Hz}, \mathrm{H}-6), 8.17(1 \mathrm{H}, \mathrm{d}, J=8.9 \mathrm{~Hz}, \mathrm{H}-5), 8.18(1 \mathrm{H}$, $\left.\mathrm{s}, \mathrm{H}-3^{\prime}\right), 8.88\left(1 \mathrm{H}, \mathrm{s}, \mathrm{H}-5^{\prime}\right) .{ }^{13} \mathrm{C}-\mathrm{NMR}\left(\mathrm{CDCl}_{3}, 400 \mathrm{MHz}\right) \delta$ : 56.0, 98.1, 100.4, 115.0, 117.3, 127.5, 141.8, 152.6, 153.9, 155.7, 164.6, 177.0. MS-EI m/z: $243\left[\mathrm{M}^{+}\right]$. Anal. Calcd for $\mathrm{C}_{12} \mathrm{H}_{9} \mathrm{~N}_{3} \mathrm{O}_{3}$ : C, 59.26; H, 3.73; N, 17.28. Found: C, 59.27; H, 3.67; N, 17.29.

(1H-1,2,3-Triazol-1-yl)-4H-1-benzopyran-4-one (5a) Yield $47 \%$. White needle crystal. mp $146^{\circ} \mathrm{C}$. IR $(\mathrm{KBr}) \mathrm{cm}^{-1}: 756$, 864, 948, 1273, 1465, 1658. ${ }^{1} \mathrm{H}-\mathrm{NMR}\left(\mathrm{CDCl}_{3}, 400 \mathrm{MHz}\right) \delta$ : $7.06(1 \mathrm{H}, \mathrm{s}, \mathrm{H}-3), 7.51$ (1H, ddd, $J=8.0,7.1,1.1 \mathrm{~Hz}, \mathrm{H}-6), 7.69$ $(1 \mathrm{H}, \mathrm{dd}, J=8.5,1.1 \mathrm{~Hz}, \mathrm{H}-8), 7.77(1 \mathrm{H}, \mathrm{ddd}, J=8.5,7.1,1.7 \mathrm{~Hz}$, H-7), 8.02 (2H, s, H-4', 5'), 8.27 (1H, dd, $J=8.0,1.7 \mathrm{~Hz}, \mathrm{H}-5)$. ${ }^{13} \mathrm{C}-\mathrm{NMR}\left(\mathrm{CDCl}_{3}, 100 \mathrm{MHz}\right) \delta: 98.8,118.3,124.0,126.2$, 126.4, 134.5, 138.5 (2C), 154.5, 154.7, 178.2. MS-EI m/z: 213 $\left[\mathrm{M}^{+}\right.$]. Anal. Calcd for $\mathrm{C}_{11} \mathrm{H}_{7} \mathrm{~N}_{3} \mathrm{O}_{2}: \mathrm{C}, 61.97 ; \mathrm{H}, 3.31 ; \mathrm{N}, 19.71$. Found: C, 61.95; H, 3.24; N 19.62.

6-Methoxy-2-(1H-1,2,3-triazol-1-yl)-4H-1-benzopyran4-one (5b) Yield 16\%. Pale yellow needle crystal. mp 216-217 ${ }^{\circ} \mathrm{C}$. IR (KBr) cm $\mathrm{cm}^{-1}$ : 825, 948, 1273, 1458, 1651, 3086. ${ }^{1} \mathrm{H}-\mathrm{NMR}\left(\mathrm{CDCl}_{3}, 400 \mathrm{MHz}\right) \delta: 3.94(3 \mathrm{H}, \mathrm{s}, \mathrm{OMe}), 7.04(1 \mathrm{H}, \mathrm{s}$, $\mathrm{H}-3), 7.34$ (1H, dd, $J=9.1,3.1 \mathrm{~Hz}, \mathrm{H}-7), 7.62(1 \mathrm{H}, \mathrm{d}, J=9.1 \mathrm{~Hz}$, H-8), 7.63 (1H, d, J=3.1 Hz, H-5), 8.01 (2H, s, H-4', 5'). ${ }^{13} \mathrm{C}-\mathrm{NMR}\left(\mathrm{CDCl}_{3}, 100 \mathrm{MHz}\right) \delta: 56.2,98.1,105.6,119.7,124.2$, 124.7, 138.4 (2C), 149.3, 154.3, 157.8, 178.1. MS-EI $\mathrm{m} / \mathrm{z}: 243$ $\left[\mathrm{M}^{+}\right]$. Anal. Calcd for $\mathrm{C}_{12} \mathrm{H}_{9} \mathrm{~N}_{3} \mathrm{O}_{3}: \mathrm{C}, 59.26 ; \mathrm{H}, 3.73 ; \mathrm{N}, 17.28$. Found: C, 59.19; H, 3.74; N, 17.17.

7-Methoxy-2-(1H-1,2,3-triazol-1-yl)-4H-1-benzopyran4-one (5c) Yield 37\%. White solid.. mp $191^{\circ} \mathrm{C}$. IR ( $\left.\mathrm{KBr}\right)$ $\mathrm{cm}^{-1}:$ 833, 910, 956, 1273, 1427, 1651, 3086. ${ }^{1} \mathrm{H}-\mathrm{NMR}\left(\mathrm{CDCl}_{3}\right.$, $400 \mathrm{MHz}) \delta: 3.94(3 \mathrm{H}, \mathrm{s}, \mathrm{OMe}), 6.99(1 \mathrm{H}, \mathrm{s}, \mathrm{H}-3), 7.06(1 \mathrm{H}, \mathrm{dd}$, $J=8.8,2.3 \mathrm{~Hz}, \mathrm{H}-6), 7.10(1 \mathrm{H}, \mathrm{dd}, J=2.3 \mathrm{~Hz}, \mathrm{H}-8), 8.00(2 \mathrm{H}$, $\left.\mathrm{s}, \mathrm{H}-4^{\prime}, 5^{\prime}\right), 8.16(1 \mathrm{H}, \mathrm{d}, J=8.8 \mathrm{~Hz}, \mathrm{H}-5) .{ }^{13} \mathrm{C}-\mathrm{NMR}\left(\mathrm{CDCl}_{3}\right.$, $100 \mathrm{MHz}) \delta: 55.9,98.4,100.4,115.2,117.3,127.1,138.0(2 \mathrm{C})$, 153.9, 156.1, 164.4, 177.3. MS-EI $m / z: 243\left[\mathrm{M}^{+}\right]$. Anal. Calcd for $\mathrm{C}_{12} \mathrm{H}_{9} \mathrm{~N}_{3} \mathrm{O}_{3}$ : C, 59.26; H, 3.73; N, 17.28. Found: C, 59.29; H, $3.66 ; \mathrm{N}, 17.29$.

2-(1H-Indol-1-yl)-4H-1-benzopyran-4-one (6a) Yield 44\%. Pale yellow needle crystal. mp $157-158^{\circ} \mathrm{C}$. IR $(\mathrm{KBr}) \mathrm{cm}^{-1}$ : 732, 1419, 1543, 1635, 3062, 3116. ${ }^{1} \mathrm{H}-\mathrm{NMR}\left(\mathrm{CDCl}_{3}, 400 \mathrm{MHz}\right)$ $\delta: 6.47(1 \mathrm{H}, \mathrm{s}, \mathrm{H}-3), 6.81\left(1 \mathrm{H}, \mathrm{d}, J=3.6 \mathrm{~Hz}, \mathrm{H}-3^{\prime}\right), 7.30(1 \mathrm{H}$, ddd, $\left.J=7.8,7.2,1.0 \mathrm{~Hz}, \mathrm{H}-5^{\prime}\right), 7.39(1 \mathrm{H}, \mathrm{ddd}, J=8.4,7.2$, $\left.1.2 \mathrm{~Hz}, \mathrm{H}-6^{\prime}\right), 7.48(1 \mathrm{H}, \mathrm{ddd}, J=7.9,7.2,1.0 \mathrm{~Hz}, \mathrm{H}-6), 7.56(1 \mathrm{H}$, d, $\left.J=3.6 \mathrm{~Hz}, \mathrm{H}-2^{\prime}\right), 7.59(1 \mathrm{H}, \mathrm{dd}, J=8.4,1.0 \mathrm{~Hz}, \mathrm{H}-8), 7.67(1 \mathrm{H}$, brd, $\left.J=7.8 \mathrm{~Hz}, \mathrm{H}-4^{\prime}\right), 7.74(1 \mathrm{H}, \mathrm{ddd}, J=8.4,7.2,1.7 \mathrm{~Hz}, \mathrm{H}-7)$, $8.01\left(1 \mathrm{H}\right.$, brd, $\left.J=8.4 \mathrm{~Hz}, \mathrm{H}^{-} 7^{\prime}\right), 8.26(1 \mathrm{H}, \mathrm{dd}, J=7.9,1.7 \mathrm{~Hz}$, H-5). ${ }^{13} \mathrm{C}-\mathrm{NMR}\left(\mathrm{CDCl}_{3}, 100 \mathrm{MHz}\right) \delta: 97.4,108.9,113.4,117.4$, $121.8,123.0,123.5,124.5,124.6,125.8,126.0,130.7,133.8$, 134.6, 154.5, 156.6, 178.1. MS-EI $m / z: 261\left[\mathrm{M}^{+}\right]$.

The ${ }^{1} \mathrm{H}-\mathrm{NMR}$ spectrum was similar to that previously reported. ${ }^{17)}$

2-(1H-Indol-1-yl)-6-methoxy-4H-1-benzopyran-4-one (6b) Yield $9 \%$. Pale brown solid. mp $166^{\circ} \mathrm{C}$. IR $(\mathrm{KBr}) \mathrm{cm}^{-1}$ : $817,1342,1450,1566,1635 .{ }^{1} \mathrm{H}-\mathrm{NMR}\left(\mathrm{CDCl}_{3}, 400 \mathrm{MHz}\right) \delta$ : $3.93(3 \mathrm{H}, \mathrm{s}, \mathrm{OMe}), 6.46(1 \mathrm{H}, \mathrm{s}, \mathrm{H}-3), 6.81(1 \mathrm{H}, \mathrm{d}, J=3.6 \mathrm{~Hz}$, H-3') $7.23-7.33$ (2H, m, H-7, 5'), 7.39 (1H, ddd, $J=8.4$,
7.2, $\left.1.7 \mathrm{~Hz}, \mathrm{H}-6^{\prime}\right), 7.52(1 \mathrm{H}, \mathrm{d}, J=9.1 \mathrm{~Hz}, \mathrm{H}-8), 7.55(1 \mathrm{H}, \mathrm{d}$, $\left.J=3.6 \mathrm{~Hz}, \mathrm{H}-2^{\prime}\right), 7.64(1 \mathrm{H}, \mathrm{d}, J=3.1 \mathrm{~Hz}, \mathrm{H}-5), 7.67(1 \mathrm{H}, \mathrm{brd}$, $\left.J=7.8 \mathrm{~Hz}, \mathrm{H}-4^{\prime}\right), 8.00\left(1 \mathrm{H}\right.$, brd, $\left.J=8.4 \mathrm{~Hz} \mathrm{H}-7^{\prime}\right) .{ }^{13} \mathrm{C}-\mathrm{NMR}$ $\left(\mathrm{CDCl}_{3}, 100 \mathrm{MHz}\right) \delta: 56.0,96.9,105.5,108.8,113.3,118.7$, $121.8,122.9,123.4,124.1,124.4,124.5,130.7,134.6,149.0$, 156.5, 157.4, 178.0. MS-EI $\mathrm{m} / z$ : $291\left[\mathrm{M}^{+}\right]$. Anal. Calcd for $\mathrm{C}_{18} \mathrm{H}_{13} \mathrm{NO}_{3}$ : C, 74.22; H, 4.50; N, 4.81. Found: C, 73.94; H, $4.41 ; \mathrm{N}, 4.70$.

2-(1H-Indol-1-yl)-7-methoxy-4H-1-benzopyran-4-one (6c) Yield $17 \%$. Pale brown solid. mp $172-174^{\circ} \mathrm{C}$. IR (KBr) $\mathrm{cm}^{-1}:$ 732, 833, 1342, 1419, 1627, 3070, 3116. ${ }^{1} \mathrm{H}-\mathrm{NMR}\left(\mathrm{CDCl}_{3}\right.$, $400 \mathrm{MHz}) \delta: 3.97(3 \mathrm{H}, \mathrm{s}, \mathrm{OMe}), 6.41(1 \mathrm{H}, \mathrm{s}, \mathrm{H}-3), 6.81(1 \mathrm{H}$, d, $\left.J=3.6 \mathrm{~Hz}, \mathrm{H}-3^{\prime}\right), 6.99(1 \mathrm{H}, \mathrm{d}, J=2.3 \mathrm{~Hz}, \mathrm{H}-8), 7.05(1 \mathrm{H}, \mathrm{dd}$, $J=8.8,2.3 \mathrm{~Hz}, \mathrm{H}-6), 7.30$ (1H, ddd, $\left.J=7.8,7.2,1.0 \mathrm{~Hz}, \mathrm{H}-5^{\prime}\right)$, $7.40\left(1 \mathrm{H}, \mathrm{ddd}, J=8.4,7.2,1.2 \mathrm{~Hz}, \mathrm{H}-6^{\prime}\right), 7.56(1 \mathrm{H}, \mathrm{d}, J=3.6 \mathrm{~Hz}$, H-2' $), 7.68\left(1 \mathrm{H}\right.$, brd, $\left.J=7.8 \mathrm{~Hz}, \mathrm{H}-4^{\prime}\right), 7.98(1 \mathrm{H}$, brd, $J=8.4 \mathrm{~Hz}$ $\left.\mathrm{H}-7^{\prime}\right), \quad 8.18(1 \mathrm{H}, \mathrm{d}, J=8.8 \mathrm{~Hz}, \mathrm{H}-5) .{ }^{13} \mathrm{C}-\mathrm{NMR} \quad\left(\mathrm{CDCl}_{3}\right.$, $100 \mathrm{MHz}) \delta$ : 56.0, 97.5, 100.4, 108.6, 113.2, 114.4, 117.1, 121.8, $122.9,124.4,124.6,127.3,130.6,134.6,156.1,156.3,164.2$, 177.7. MS-EI $m / z: 291\left[\mathrm{M}^{+}\right]$. Anal. Calcd for $\mathrm{C}_{18} \mathrm{H}_{13} \mathrm{NO}_{3}$ : C, 74.22; H, 4.50; N, 4.81. Found: C, 73.97; H, 4.47; N, 4.81.

2-(1H-Indazol-1-yl)-4H-1-benzopyran-4-one (7a) Yield $89 \%$. White needle crystal. mp $239-240^{\circ} \mathrm{C}$. IR (KBr) $\mathrm{cm}^{-1}$ : $748,1180,1419,1566,1627,3055 .{ }^{1} \mathrm{H}-\mathrm{NMR}\left(\mathrm{CDCl}_{3}, 400 \mathrm{MHz}\right)$ $\delta: 6.95(1 \mathrm{H}, \mathrm{s}, \mathrm{H}-3), 7.39\left(1 \mathrm{H}, \mathrm{ddd}, J=8.0,7.0,0.9 \mathrm{~Hz}, \mathrm{H}-5^{\prime}\right)$, $7.50(1 \mathrm{H}$, ddd, $J=8.0,7.0,1.1 \mathrm{~Hz}, \mathrm{H}-6), 7.63$ (1H, ddd, $J=8.6$, 7.0, $\left.1.1 \mathrm{~Hz}, \mathrm{H}-6^{\prime}\right), 7.67(1 \mathrm{H}, \mathrm{dd}, J=8.5,1.1 \mathrm{~Hz}, \mathrm{H}-8), 7.76(1 \mathrm{H}$, ddd, $J=8.5,7.1,1.7 \mathrm{~Hz}, \mathrm{H}-7), 7.84(1 \mathrm{H}, \mathrm{dt}, J=8.0,1.0 \mathrm{~Hz}$, $\left.\mathrm{H}-4^{\prime}\right), 8.27\left(1 \mathrm{H}\right.$, brd, $\left.J=8.6 \mathrm{~Hz}, \mathrm{H}-7^{\prime}\right), 8.30(1 \mathrm{H}, \mathrm{dd}, J=8.0$, $1.7 \mathrm{~Hz}, \mathrm{H}-5), 8.32\left(1 \mathrm{H}, \mathrm{d}, J=0.9 \mathrm{~Hz}, \mathrm{H}-3^{\prime}\right) \cdot{ }^{13} \mathrm{C}-\mathrm{NMR}\left(\mathrm{CDCl}_{3}\right.$, $100 \mathrm{MHz}) \delta: 96.5,113.5,117.2,121.8,123.8,123.9,125.9$, $126.2,126.5,129.2,133.6,138.6,140.3,154.0,158.0,177.8$. MS-EI $m / z: 262\left[\mathrm{M}^{+}\right]$.

The ${ }^{1} \mathrm{H}-\mathrm{NMR}$ spectrum was similar to that previously reported. ${ }^{17)}$

2-(1H-Indazol-1-yl)-6-methoxy-4H-1-benzopyran-4-one (7b) Yield $75 \%$. White solid. mp $206-208^{\circ} \mathrm{C}$. IR (KBr) $\mathrm{cm}^{-1}$ : 756, 1018, 1573, 3078. ${ }^{1} \mathrm{H}-\mathrm{NMR}\left(\mathrm{CDCl}_{3}, 400 \mathrm{MHz}\right) \delta: 3.94$ $(3 \mathrm{H}, \mathrm{s}, \mathrm{OMe}), 6.94(1 \mathrm{H}, \mathrm{s}, \mathrm{H}-3), 7.32(1 \mathrm{H}, \mathrm{dd}, J=9.0,3.1 \mathrm{~Hz}$, H-7), 7.39 (1H, ddd, $\left.J=8.0,7.0,0.9 \mathrm{~Hz}, \mathrm{H}-5^{\prime}\right), 6.59(1 \mathrm{H}, \mathrm{d}$, $J=9.0 \mathrm{~Hz}, \mathrm{H}-8), 7.62\left(1 \mathrm{H}\right.$, ddd, $\left.J=8.6,7.0,1.1 \mathrm{~Hz}, \mathrm{H}-6^{\prime}\right), 7.68$ $(1 \mathrm{H}, \mathrm{d}, J=3.1 \mathrm{~Hz}, \mathrm{H}-5), 7.83\left(1 \mathrm{H}, \mathrm{dt}, J=8.0,1.0 \mathrm{~Hz}, \mathrm{H}-4^{\prime}\right)$, $8.25\left(1 \mathrm{H}\right.$, brd, $\left.J=8.6 \mathrm{~Hz}, \mathrm{H}-7^{\prime}\right), 8.31\left(1 \mathrm{H}, \mathrm{d}, J=0.9 \mathrm{~Hz}, \mathrm{H}-3^{\prime}\right)$. ${ }^{13} \mathrm{C}-\mathrm{NMR}\left(\mathrm{CDCl}_{3}, 100 \mathrm{MHz}\right) \delta: 56.0,95.9,105.7,113.4,118.6$, $121.8,123.2,123.8,124.6,126.4,129.1,138.6,140.2,148.6$, 157.9, 157.8, 177.7. MS-EI $m / z: 292\left[\mathrm{M}^{+}\right]$. Anal. Calcd for $\mathrm{C}_{17} \mathrm{H}_{12} \mathrm{~N}_{2} \mathrm{O}_{3}$ : C, 69.86; H, 4.14; N, 9.58. Found: C, 69.85; H, $4.01 ; \mathrm{N}, 9.48$.

2-(1H-Indazol-1-yl)-7-methoxy-4H-1-benzopyran-4-one (7c) Yield $75 \%$. White solid. mp $219-222^{\circ} \mathrm{C}$. IR $(\mathrm{KBr}) \mathrm{cm}^{-1}$ : 740, 1010, 1165, 1419, 1566, 3070. ${ }^{1} \mathrm{H}-\mathrm{NMR}\left(\mathrm{CDCl}_{3}, 400 \mathrm{MHz}\right)$ $\delta: 3.99$ (3H, s, OMe), 6.86 (1H, s, H-3), 7.03-7.07 (2H, m, H-6, 8), $7.39\left(1 \mathrm{H}, \mathrm{ddd}, J=8.0,7.0,0.8 \mathrm{~Hz}, \mathrm{H}-5^{\prime}\right), 7.63(1 \mathrm{H}$, ddd, $\left.J=8.6,7.0,1.1 \mathrm{~Hz}, \mathrm{H}-6^{\prime}\right), 7.83\left(1 \mathrm{H}, \mathrm{dt}, J=8.0,1.0 \mathrm{~Hz}, \mathrm{H}-4^{\prime}\right)$, $8.19(1 \mathrm{H}, \mathrm{d}, J=8.6 \mathrm{~Hz}, \mathrm{H}-5), 8.21\left(1 \mathrm{H}, \mathrm{brd}, J=8.6 \mathrm{~Hz}, \mathrm{H}-7^{\prime}\right)$, $8.31\left(1 \mathrm{H}, \mathrm{d}, J=0.9 \mathrm{~Hz}, \mathrm{H}-3^{\prime}\right) .{ }^{13} \mathrm{C}-\mathrm{NMR}\left(\mathrm{CDCl}_{3}, 100 \mathrm{MHz}\right) \delta$ : 56.0, 96.5, 100.4, 113.3, 114.3, 117.6, 121.8, 123.8, 126.4, 127.4, 129.1, 138.5, 140.0, 155.7, 157.6, 164.0, 177.4. MS-EI m/z: 292 $\left[\mathrm{M}^{+}\right.$]. Anal. Calcd for $\mathrm{C}_{17} \mathrm{H}_{12} \mathrm{~N}_{2} \mathrm{O}_{3}:$ C, 69.86; H, 4.14; N, 9.58. Found: C, 69.57; H, 4.00; N, 9.41.

2-(1H-Benzimidazol-1-yl)-4H-1-benzopyran-4-one 
Yield $48 \%$. White crystal. mp $232-234^{\circ} \mathrm{C}$. IR (KBr) cm ${ }^{-1}: 617$, 748, 1118, 1180, 1311, 1411, 1651, 3070, 3109. ${ }^{1} \mathrm{H}-\mathrm{NMR}\left(\mathrm{CDCl}_{3}\right.$, $400 \mathrm{MHz}) \delta: 6.60(1 \mathrm{H}, \mathrm{s}, \mathrm{H}-3), 7.43-7.53\left(2 \mathrm{H}, \mathrm{m}, \mathrm{H}-5^{\prime}, 6^{\prime}\right), 7.53$ $(1 \mathrm{H}$, ddd, $J=7.9,7.1,1.0 \mathrm{~Hz}, \mathrm{H}-6), 7.62(1 \mathrm{H}, \mathrm{dd}, J=8.4,1.0 \mathrm{~Hz}$, $\mathrm{H}-8), 7.79$ (1H, ddd, $J=8.4,7.1,1.7 \mathrm{~Hz}, \mathrm{H}-7), 7.87-7.94(2 \mathrm{H}, \mathrm{m}$, H-4', 7'), 8.29 (1H, dd, J=7.9, $1.7 \mathrm{~Hz}, \mathrm{H}-5), 8.48$ (1H, s, H-2').

${ }^{13} \mathrm{C}-\mathrm{NMR}\left(\mathrm{CDCl}_{3}, 100 \mathrm{MHz}\right) \delta: 98.9,112.4,117.6,121.5,123.5$, $124.7,125.5,126.1,126.3,131.0,134.3,139.8,144.4,153.9$, 154.5, 177.8. MS-EI $m / z: 262\left(\mathrm{M}^{+}\right)$.

The ${ }^{1} \mathrm{H}-\mathrm{NMR}$ spectrum was similar to that previously reported. ${ }^{17)}$

2-(1H-Benzimidazol-1-yl)-6-methoxy-4H-1-benzopyran4-one (8b) Yield 54\%. White crystal. mp $221^{\circ} \mathrm{C}$. IR (KBr) $\mathrm{cm}^{-1}:$ 894, 1018, 1404, 1573, 1620, 3001, 3078. ${ }^{1} \mathrm{H}-\mathrm{NMR}$ $\left(\mathrm{CDCl}_{3}, 400 \mathrm{MHz}\right) \delta: 3.94$ (3H, s, OMe), 6.59 (1H, s, H-3), 7.36 (1H, dd, $J=9.1,3.1 \mathrm{~Hz}, \mathrm{H}-7), 7.43-7.52$ (2H, m, H-5', 6'), 7.55 $(1 \mathrm{H}, \mathrm{d}, J=9.0 \mathrm{~Hz}, \mathrm{H}-8), 7.65(1 \mathrm{H}, \mathrm{d}, J=3.1 \mathrm{~Hz}, \mathrm{H}-5), 7.87-7.94$ $\left(2 \mathrm{H}, \mathrm{m}, \mathrm{H}-4^{\prime}, 7^{\prime}\right), 8.46\left(1 \mathrm{H}, \mathrm{s}, \mathrm{H}-2^{\prime}\right) .{ }^{13} \mathrm{C}-\mathrm{NMR}\left(\mathrm{CDCl}_{3}\right.$, $100 \mathrm{MHz}) \delta: 56.0,98.3,105.6,112.4,118.9,121.4,124.0,124.2$, 124.7, 125.5, 131.0, 139.8, 144.4, 149.1, 153.7, 157.7, 177.8. MS-EI m/z: $292\left[\mathrm{M}^{+}\right]$. Anal. Calcd for $\mathrm{C}_{17} \mathrm{H}_{12} \mathrm{~N}_{2} \mathrm{O}_{3}$ : C, 69.86; H, 4.14; N, 9.58. Found: C, 69.76; H, 4.04; N, 9.47.

2-(1H-Benzimidazol-1-yl)-7-methoxy-4H-1-benzopyran4-one (8c) Yield 54\%. White solid. mp $200^{\circ} \mathrm{C}$. IR $(\mathrm{KBr})$ $\mathrm{cm}^{-1}:$ 624, 740, 1080, 1165, 1404, 1627, 3078. ${ }^{1} \mathrm{H}-\mathrm{NMR}$ $\left(\mathrm{CDCl}_{3}, 400 \mathrm{MHz}\right) \delta: 3.97$ (3H, s, OMe), $6.53(1 \mathrm{H}, \mathrm{s}, \mathrm{H}-3)$, $6.99(1 \mathrm{H}, \mathrm{d}, J=2.3 \mathrm{~Hz}, \mathrm{H}-8), 7.07$ (1H, dd, $J=8.9,2.3 \mathrm{~Hz}, \mathrm{H}-6)$, 7.42-7.51 (2H, m, H-5', 6'), 7.84-7.93 (2H, m, H-4', 7'), 8.17 $(1 \mathrm{H}, \mathrm{d}, J=8.9 \mathrm{~Hz}, \mathrm{H}-5), 8.45\left(1 \mathrm{H}, \mathrm{s}, \mathrm{H}-2^{\prime}\right) .{ }^{13} \mathrm{C}-\mathrm{NMR}\left(\mathrm{CDCl}_{3}\right.$, $100 \mathrm{MHz}) \delta: 56.0,99.0,100.4,112.3,115.0,117.1,121.4,124.6$, $125.4,127.4,131.0,139.8,144.4,153.5,156.2,164.6,177.3$. MS-EI m/z: $292\left[\mathrm{M}^{+}\right]$. Anal. Calcd for $\mathrm{C}_{17} \mathrm{H}_{12} \mathrm{~N}_{2} \mathrm{O}_{3}$ : C; 69.86, H; 4.14, N, 9.58. Found: C, 69.66; H, 3.99; N, 9.44.

Biological Assay Recombinant human monoamine oxidase A (MAO-A), MAO-B, pargyline and kynuramine were purchased from Sigma-Aldrich Japan.

MAO Inhibitory Assay MAO inhibitory activity was assayed using the method of Novaroli et al. ${ }^{20)}$ with minor modifications. Briefly, $140 \mu \mathrm{L}$ of $0.1 \mathrm{M}$ potassium phosphate buffer ( $\mathrm{pH} 7.4$ ), $8 \mu \mathrm{L}$ of $0.75 \mathrm{~mm}$ kynuramine, and $2 \mu \mathrm{L}$ of a dimethyl sulfoxide (DMSO) inhibitor solution (final DMSO concentration of $1 \%(\mathrm{v} / \mathrm{v}))$, were preincubated at $37^{\circ} \mathrm{C}$ for $10 \mathrm{~min}$. Diluted human recombinant enzyme $(50 \mu \mathrm{L})$ was then added to obtain a final protein concentration of $0.0075 \mathrm{mg} /$ $\mathrm{mL}$ (MAO-A) or $0.015 \mathrm{mg} / \mathrm{mL}$ (MAO-B) in the assay mixture. Further incubation was carried out at $37^{\circ} \mathrm{C}$ and the reaction was stopped after $20 \mathrm{~min}$ by addition of $75 \mu \mathrm{L}$ of $2 \mathrm{M} \mathrm{NaOH}$. The product generated by MAO, 4-quinolinol, is fluorescent and was measured at Ex $310 \mathrm{~nm} / E m 400 \mathrm{~nm}$ using a microplate reader (Molecular Devices SPECTRA MAX M2). The sample solution was replaced with DMSO to provide a negative control and pargyline was used as a positive control.

Conflict of Interest The authors declare no conflict of interest.

\section{References}

1) Gaspar A., Matos M. J., Garrido J., Uriarte E., Borges F., Chem. Rev., 114, 4960-4992 (2014).

2) Gaspar A., Silva T., Yáñez M., Vina D., Orallo F., Ortuso F., Uriarte E., Alcaro S., Borges F., J. Med. Chem., 54, 5165-5173 (2011).

3) Edmondson D. E., DeColibus L., Binda C., Li M., Mattevi A., J. Neural Transm., 114, 703-705 (2007).

4) De Colibus L., Li M., Binda C., Lustig A., Edmondson D. E., Mattevi A., Proc. Natl. Acad. Sci. U.S.A., 102, 12684-12689 (2005).

5) Kalgutkar A. S., Castagnoli N. Jr., Testa B., Med. Res. Rev., 15, 325-388 (1995)

6) Alcaro S., Gaspar A., Ortuso F., Milhazes N., Orallo F., Uriarte E., Yáñez M., Borges F., Bioorg. Med. Chem. Lett., 20, 2709-2712 (2010).

7) Gaspar A., Reis J., Fonseca A., Milhazes N., Viña D., Uriarte E., Borges F., Bioorg. Med. Chem. Lett., 21, 707-709 (2011).

8) Cagide F., Silva T., Reis J., Gaspar A., Borges F., Gomes L. R., Low J. N., Chem. Commun., 51, 2832-2835 (2015).

9) Reis J., Cagide F., Chavarria D., Silva T., Fernandes C., Gaspar A., Uriarte E., Remião F., Alcaro S., Ortuso F., Borges F., J. Med. Chem., 59, 5879-5893 (2016).

10) Legoabe L. J., Petzer A., Petzer J. P., Bioorg. Med. Chem. Lett., 22, 5480-5484 (2012).

11) Legoabe L. J., Petzer A., Petzer J. P., Eur. J. Med. Chem., 49, 343353 (2012).

12) Legoabe L. J., Petzer A., Petzer J. P., Bioorg. Chem., 45, 1-11 (2012).

13) La Regina G., Silvestri R., Artico M., Lavecchia A., Novellino E., Befani O., Turini P., Agostinelli E., J. Med. Chem., 50, 922-931 (2007).

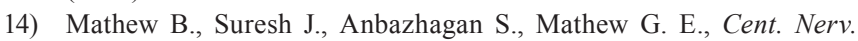
Syst. Agents Med. Chem., 13, 195-206 (2014).

15) Prins L. H., Petzer J. P., Malan S. F., Eur. J. Med. Chem., 45, 44584466 (2010).

16) Grandi T., Sparatore F., Gnerre C., Crivori P., Carrupt P. A., Testa B., AAPS PharmSci, 1, E16 (1999).

17) Sugita Y., Yokoe I., Heterocycles, 43, 2503-2511 (1996).

18) Vasselin D. A., Westwell A. D., Matthews C. S., Bradshaw T. D., Stevens M. F., J. Med. Chem., 49, 3973-3981 (2006).

19) Samanta R., Narayan R., Bauer J. O., Strohmann C., Sievers S., Antonchick A. P., Chem. Commun., 51, 925-928 (2015).

20) Novaroli L., Reist M., Favre E., Carotti A., Catto M., Carrupt P. A., Bioorg. Med. Chem., 13, 6212-6217 (2005). 